\title{
Casos de estudio de nieblas de radiación en zonas de las cuencas hidrográficas del Tajo y del Guadiana. Evaluación de observaciones y simulaciones del modelo HARMONIE-AROME v. c40
}

\author{
Guadalupe Sáenz García ${ }^{1}$ (gsaenzg@aemet.es) \\ Manuel Lara Jaén'1 (mlaraj@aemet.es) \\ Marcelino Núñez Corchero'1 (mnunezc@aemet.es)
}

${ }^{1}$ AEMET / Delegación Territorial en Extremadura

\begin{abstract}
RESUMEN
Las nieblas producen una disminución de la visibilidad que afecta seriamente a la seguridad en el transporte entre otras actividades de interés. Sin embargo, los procesos que influyen sobre ellas, y en la formación y la disipación de las mismas, no están bien resueltos en los modelos de predicción numérica en la actualidad.

Con este trabajo se ha querido dar un paso más en el análisis de la formación, desarrollo y disipación de nieblas de radiación alrededor de las cuencas hidrográficas del Tajo y el Guadiana. Para ello, se han estudiado varios casos de nieblas de radiación en esta región a partir de observaciones puntuales de visibilidad de estaciones automáticas, obteniéndose grandes discrepancias. También, se ha evaluado espacial y temporalmente el desarrollo y distribución del campo de nubes bajas previstas por el modelo no hidrostático HARMONIE-AROME (H-A) con las observadas en las imágenes del satélite Meteosat, encontrándose en general una tendencia de infraestimación durante la fase de disipación.
\end{abstract}

PALABRAS CLAVE: nieblas de radiación; verificación; HARMONIE-AROME; predicción numérica; Meteosat.

\section{INTRODUCCIÓN}

La comunidad científica ha mostrado un gran interés en el conocimiento de las nieblas a lo largo de las últimas décadas debido a sus efectos en las actividades humanas. Sin embargo, existe aún un conocimiento incompleto de estos procesos desde escalas temporales a espaciales, ya que involucran diferentes y complicados procesos físicos.

Con este trabajo preliminar se ha querido realizar un pequeño acercamiento a estos fenómenos a través de casos de estudios en un área limitada, como son las cuencas hidrográficas del Tajo y el Guadiana con condiciones homogéneas del terreno y donde aparecen usualmente nieblas de radiación durante el invierno. En primer momento, se han inspeccionado las observaciones de temperatura en superficie, humedad relativa, viento en superficie y visibilidad disponibles de la red de estaciones automáticas de AEMET localizadas en la región de estudio. A partir de ellas y de las salidas del modelo H-A cada 15 minutos se ha realizado un análisis comparativo estadístico tradicional con parámetros como el bias y el RMSE para la evaluación de 3 casos de estudios de nieblas de radiación desarrollados durante enero de 2018. 
Considerando las conocidas limitaciones de estos esquemas de verificación basados en puntos de rejilla, se ha explorado el uso de un método de verificación basado en objetos para la comparación espacial y temporal de este fenómeno usando productos de satélite del SAF de Nowcasting (NWC SAF) de EUMETSAT para verificar las salidas del modelo $\mathrm{H}-\mathrm{A}$.

\section{DATOS Y METODOLOGÍA}

En este estudio se han utilizado tres tipos de datos:

- Campos de visibilidad y cobertura de nubes bajas (Low Cloud Cover) simuladas por el modelo de predicción no hidrostático H-A versión c40 con resolución de 2,5 km (SEITY et al., 2011).

- Observaciones de temperatura en superficie $\left({ }^{\circ} \mathrm{C}\right)$, humedad relativa $(\%)$, viento en superficie $(\mathrm{m} / \mathrm{s})$ y visibilidad $(\mathrm{m})$ de estaciones automáticas representadas en la figura 1 . Se han elegido estas estaciones porque se encuentran localizadas en los lugares idóneos para la formación de nieblas de radiación durante los meses de otoño e invierno.

También se usaron medidas adicionales de visibilidad extraídas de los partes METAR del aeropuerto de Talavera la Real.

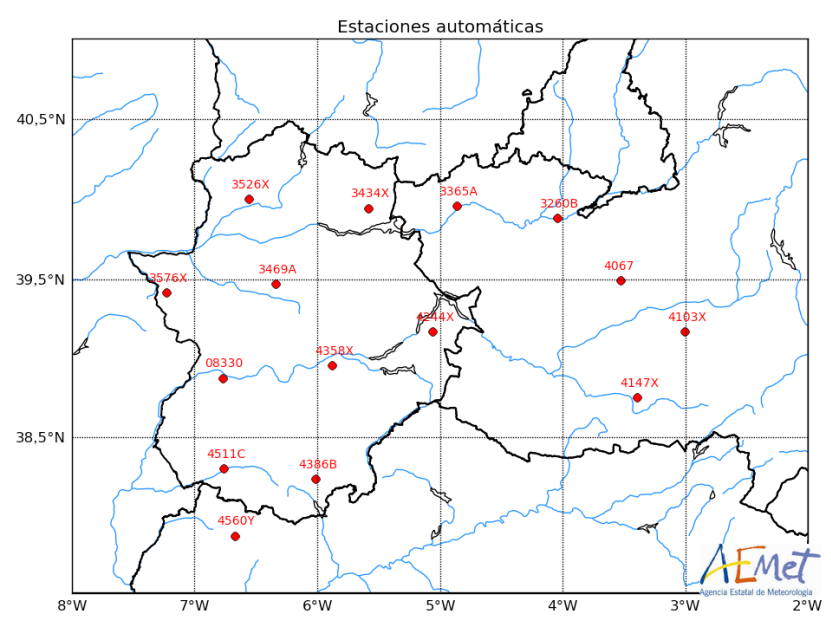

Figura 1. Mapa con la localización de las estaciones automáticas utilizadas en este estudio alrededor de las cuencas hidrográficas del Guadiana y el Tajo.

- Imágenes y productos de satélite en particular, el producto tipo de nube (Cloud Type) del NWC SAF de EUMESAT con resolución de 3 km en el nadir.

Para llevar a cabo la verificación y comparación de las estimaciones del modelo con las observaciones se ha realizado la lectura tanto de las salidas del modelo como de las imágenes y productos del satélite en los puntos en los que se localizan las estaciones automáticas. El periodo de estudio se ciñe únicamente a los episodios de nieblas que se han producido entre los días 20 y 25 de enero de 2018.

Para la comparación espacial y temporal de los productos de satélite con las salidas del modelo H-A, se han leído los datos del producto tipo de nube (Cloud Type) del SAF en formato HDF-5, filtrándolos y normalizándolos a los valores de nubes bajas y muy bajas. Esta lectura se ha realizado usando el paquete pytroll de Python para la lectura de ficheros de satélite en formato nativo. Un ejemplo de ambos conjuntos de datos una vez procesados puede verse en la figura 2.
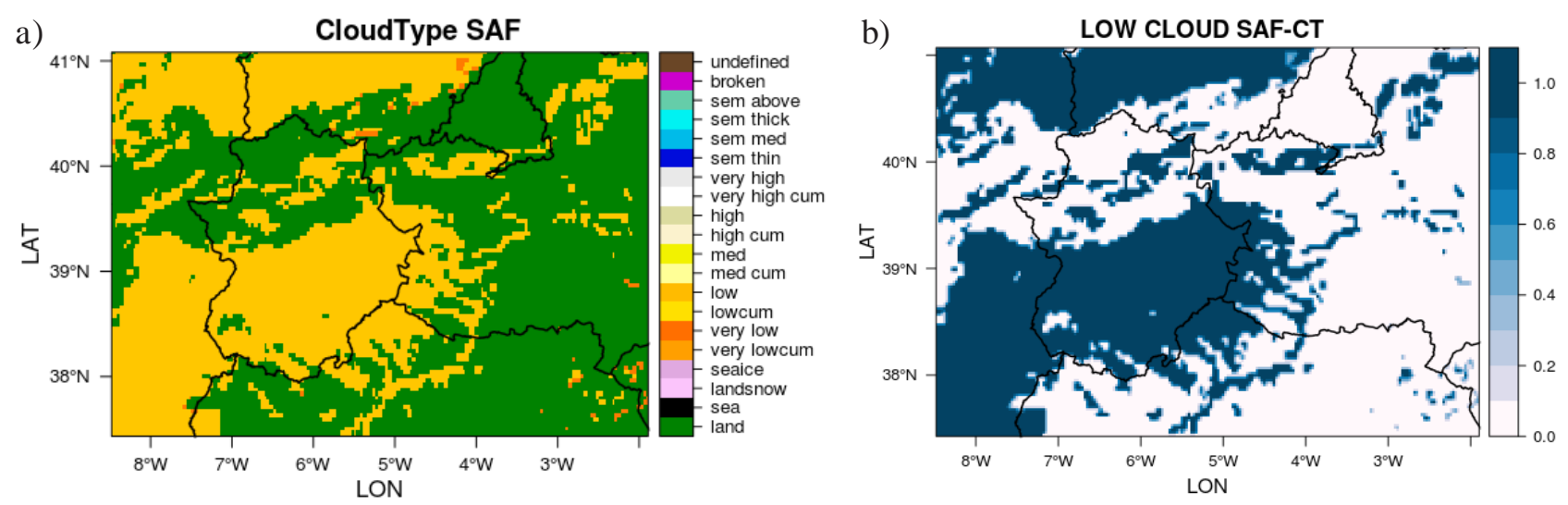

Figura 2. a) Producto CT (Cloud Type) del SAF para la región de Extremadura y

b) el mismo producto una vez filtrado y normalizado para los valores de nubes bajas y muy bajas. 
Por otro lado, el campo de nubes bajas del modelo H-A que se encuentra en formato GRIB1 se ha leído a través del paquete pygrib de Python (ejemplo, véase figura 3 ).

Por último, para la comparación de ambos conjuntos de datos se han transformado al mismo grid y a la misma escala entre 0 y 1 (véase figura $2 . b$, una vez proyectado y filtrado el campo).

Finalmente, para la comparación espacial y temporal de los productos de satélite y la predicción del modelo se ha usado el algoritmo SAL (WENIGER et al., 2016), para ello se ha realizado un método de convolución para la identificación de objetos (regiones de interés) con un proceso de suavizado. Posteriormente, el campo convolu-

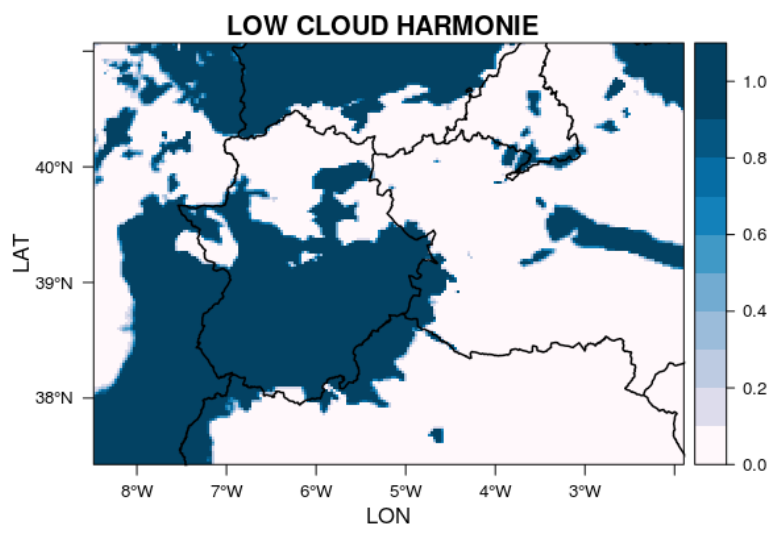

Figura 3. Campo de cobertura de nubes bajas (Low Cloud Cover) de H-A para la región de Extremadura para el mismo caso que en la figura 2 . cionado ha sido limitado por un umbral expresado en términos de percentiles, con este paso se permite detectar los límites de los objetos y estos umbrales se pueden ajustar para distinguir áreas de mayor tamaño e intensidad de aquellas que son más débiles y más aisladas. El resultado de la convolución y la aplicación de umbrales es una máscara binaria que se coloca sobre los datos originales del campo, conservándose los valores del campo dentro de cada objeto. En la figura 4 se puede ver un ejemplo de los objetos identificados en los campos de nubes bajas del producto SAF y de H-A.

a)

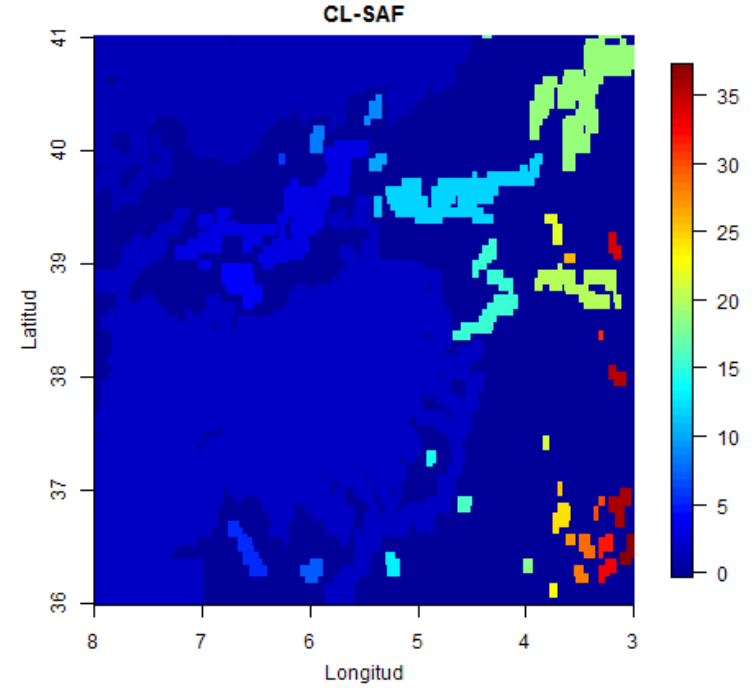

b)

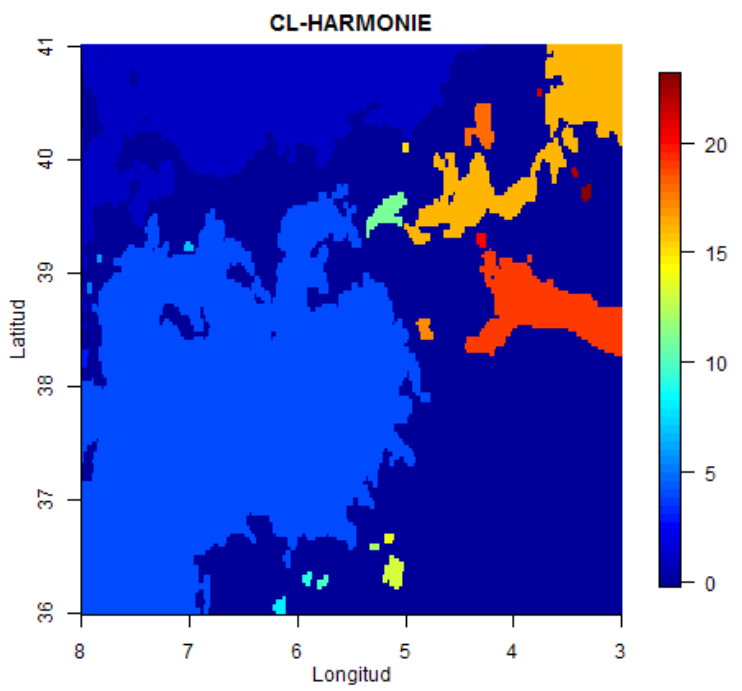

Figura 4. Objetos identificados en el campo de nubes bajas a) del SAF y

b) H-A para el mismo ejemplo presentado en las figuras 2 y 3 .

Una vez identificados los objetos en cada campo se ha aplicado el algoritmo SAL (WERNLI et al., 2008; DAvis et al., 2006). Este método calcula atributos de los objetos individuales anteriormente localizados con el método de convolución y promedia estos valores por separado para cada uno de los campos observacionales y de predicción numérica, y por último calcula las diferencias normalizadas entre los valores promedios de los campos, proporcionando los siguientes tres parámetros: $(\mathrm{S}=$ estructura) que compara la forma y el tamaño de los objetos, $(\mathrm{A}=$ amplitud $)$ que compara cuantitativamente los objetos de fracción nubosa, y (L= localización) que compara la localización de los objetos desde un modo relativo y absoluto.

Cuanto más cerca de cero estén estos parámetros mejor será la comparación. Si S > 0 y A > 0 significa una sobrestimación de las observaciones. Valores altos de L significan una errónea localización de las estructuras. 
Valores negativos de A suponen una subestimación de las observaciones. Valores grandes de S corresponden a grandes estructuras predichas por el modelo en una situación de pequeños objetos observados. Valores negativos de S significan una predicción demasiado pequeña o aguda. Para calcular el método SAL se ha utilizado el paquete libre SpatialVx, en particular la función saller, de R-cran creado en el NCAR. Con este paquete se han podido calcular los objetos con el método descrito anteriormente, a la vez que se iban visualizando, con lo que se han decidido así los mejores umbrales y valor de suavizado.

\section{RESULTADOS}

Se han seleccionado 3 casos de estudios o eventos de nieblas de radiación invernales localizados en la cuenca hidrográfica del Guadiana entre los días 21 y 24 de enero de 2018, cada evento se prolonga durante las horas nocturnas 04 UTC y hasta las 14 UTC de los días 21 (evento 1), 22 (evento 2) y 24 (evento 3) aproximadamente. Estos 3 casos se han localizado y monitorizado a partir de las imágenes del canal IR10.8 del satélite Meteosat (figura 5).

Con la inspección y comparación de las imágenes de los productos del NWC SAF y de H-A se ha observado que la mayoría de los fenómenos estudiados en las imágenes del satélite fueron reproducidos por el modelo con una cobertura espacial muy similar a la observación, aunque en las imágenes del satélite se observaban elementos más pequeños no reproducidos por el modelo. Además, también se han encontrado diferencias en la secuencia temporal de la formación y la disipación del modelo con respecto a las observaciones.

a)

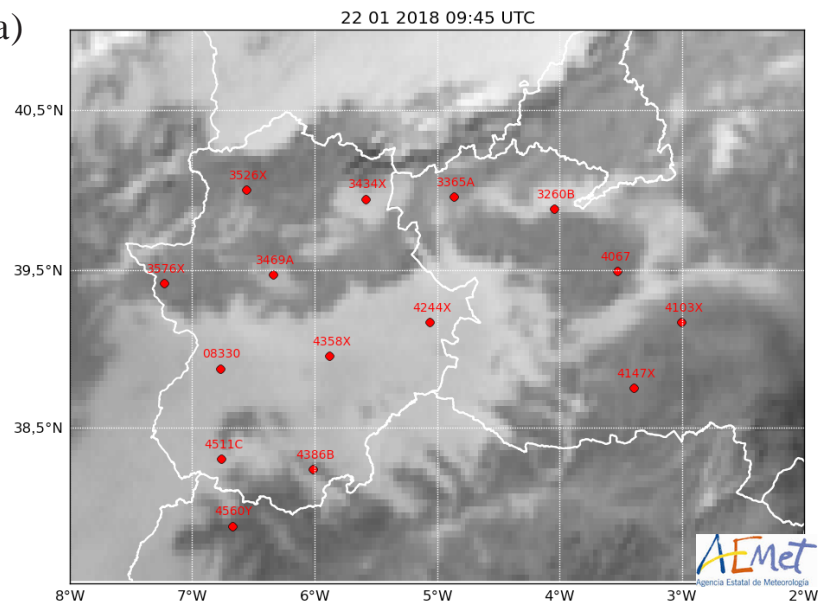

Figura 5.

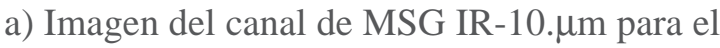
evento 2 a las 9:45, b) el campo de nubes bajas y muy bajas del producto CT del NWC SAF para el mismo evento y el mismo momento y c) el mismo caso pero la predicción de H-A. b)

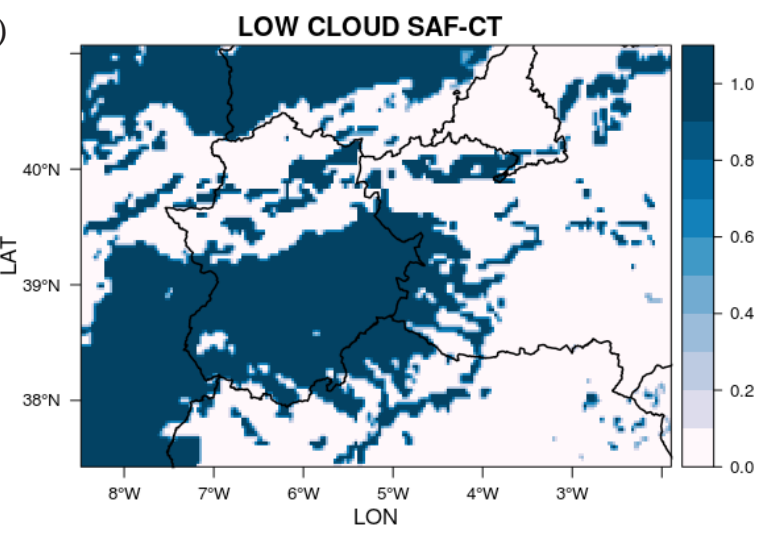

c)

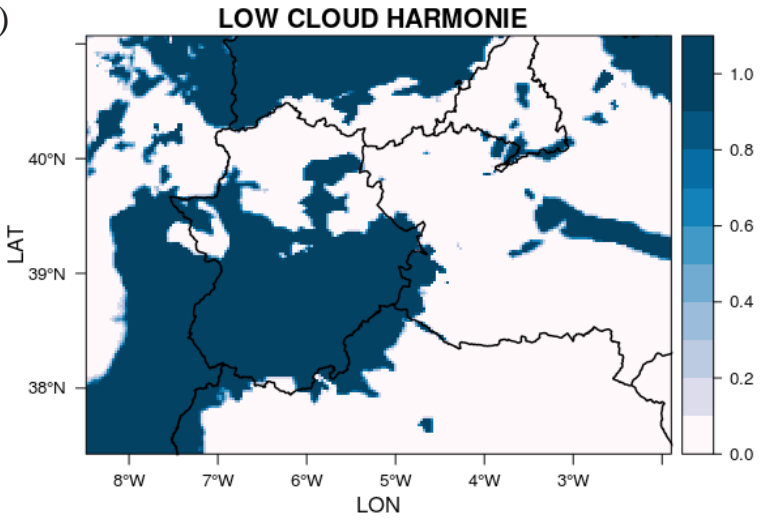

\subsection{Análisis observacional: comparación de observaciones de estaciones automáticas con las salidas del modelo $\mathrm{H}-\mathrm{A}$}

El periodo de estudio abarca del día 20 al 25 de enero de 2018. La situación sinóptica de este periodo estuvo marcada por altas presiones que afectaron a la península ibérica y que provocaron varios días con nieblas de radiación en las cuencas hidrográficas del Tajo y el Guadiana en la región de Extremadura. En un primer 
momento, se han comparado las observaciones horarias de las estaciones automáticas y del aeropuerto de Talavera la Real, de temperatura, humedad relativa, viento en superficie y visibilidad con respecto a los valores predichos para las mismas variables del modelo H-A. En la figura 6 se muestra un ejemplo de la comparación para la estación automática de Cáceres.

a)

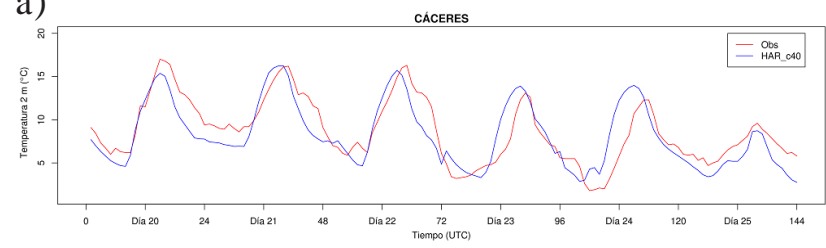

c)

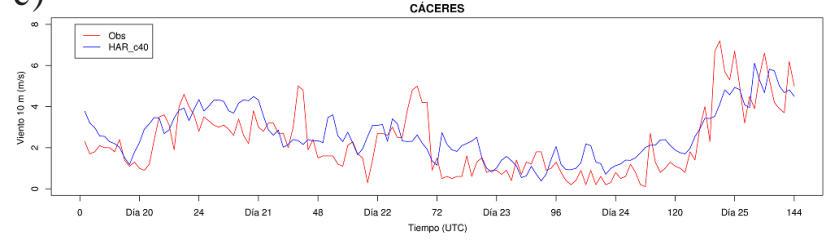

b)

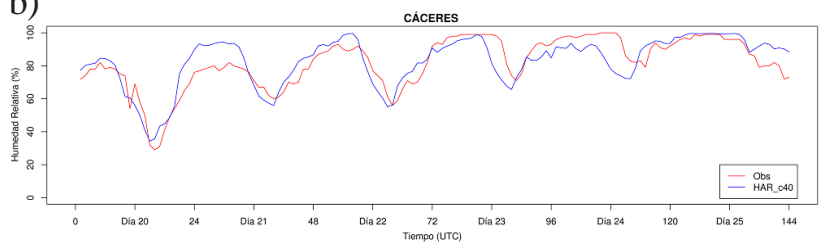

d)

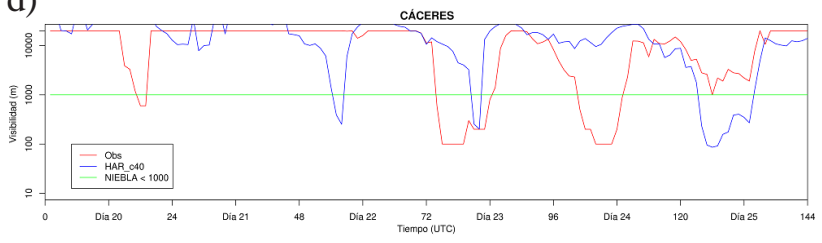

Figura 6. Ejemplo de la comparación de observaciones: a) temperatura a 2 metros, b) humedad relativa, c) viento a $10 \mathrm{~m}$ y d) visibilidad, de la estación automática de Cáceres con respecto a los valores previstos para esas mismas variables por el modelo H-A en esa misma localización.

En general, como se aprecia en este ejemplo de la figura 6 y para las demás estaciones automáticas utilizadas en este estudio (no mostrado), el acuerdo entre el modelo y las observaciones es aceptable para la variables de temperatura, humedad y viento en superficie, sin embargo en el caso de la visibilidad se aprecian mayores discrepancias.

Finalmente, se ha realizado un análisis estadístico para estas mismas variables y se han calculado las diferencias y el error cuadrático medio para el conjunto de las 15 estaciones (mostradas en la figura 1) en tres situaciones diferenciadas con respecto al proceso de nieblas: todo el periodo de estudio, solo para el periodo donde se produjeron nieblas y para el periodo sin nieblas.

\begin{tabular}{|c|c|c|c|}
\hline BIAS/RMSE & TODO EL PERIODO & CON NIEBLA & SIN NIEBLA \\
\hline TEMP 2M & $0,08 \pm 2,44$ & $0,67^{*} \pm 2,95$ & $-0,03 \pm 2,33$ \\
\hline HUMEDAD & $-1,49^{*} \pm 12,01$ & $-4,16^{*} \pm 11,14$ & $-0,98^{*} \pm 12,17$ \\
\hline VIENTO 10M & $0,38^{*} \pm 1,31$ & $0,25^{*} \pm 1,19$ & $0,41^{*} \pm 1,33$ \\
\hline VISIBILIDAD & $1226^{*} \pm 4002$ & $5336^{*} \pm 6851$ & $430^{*} \pm 3167$ \\
\hline
\end{tabular}

$\left(^{*}\right)$ significativo según el test de Student $\left(\mathrm{p}_{\text {valor }}<0,01\right)$

Tabla 1. Valores medios de bias y RMSE para la comparación de las variables de temperatura en superficie, humedad, viento en superficie y visibilidad en el conjunto de las 15 estaciones automáticas con respecto a las predicciones del modelo $\mathrm{H}-\mathrm{A}$.

Los resultados muestran mayores diferencias significativas y error cuadrático medio para el periodo con niebla y en especial en el caso de la visibilidad, como se había observado en la inspección de los datos individuales (figura 6).

Los valores altos de humedad relativa (> 95\%) (no mostrado) y la visibilidad por debajo de $1000 \mathrm{~m}$ en los informes METAR del aeropuerto de Talavera la Real (figura 7) señalan la ocurrencia de nieblas durante los siguientes días del periodo de estudio 21, 22 y 24 de enero de 2018. Estas nieblas estuvieron asociadas a fuertes inversiones térmicas superficiales y vientos bajos. 


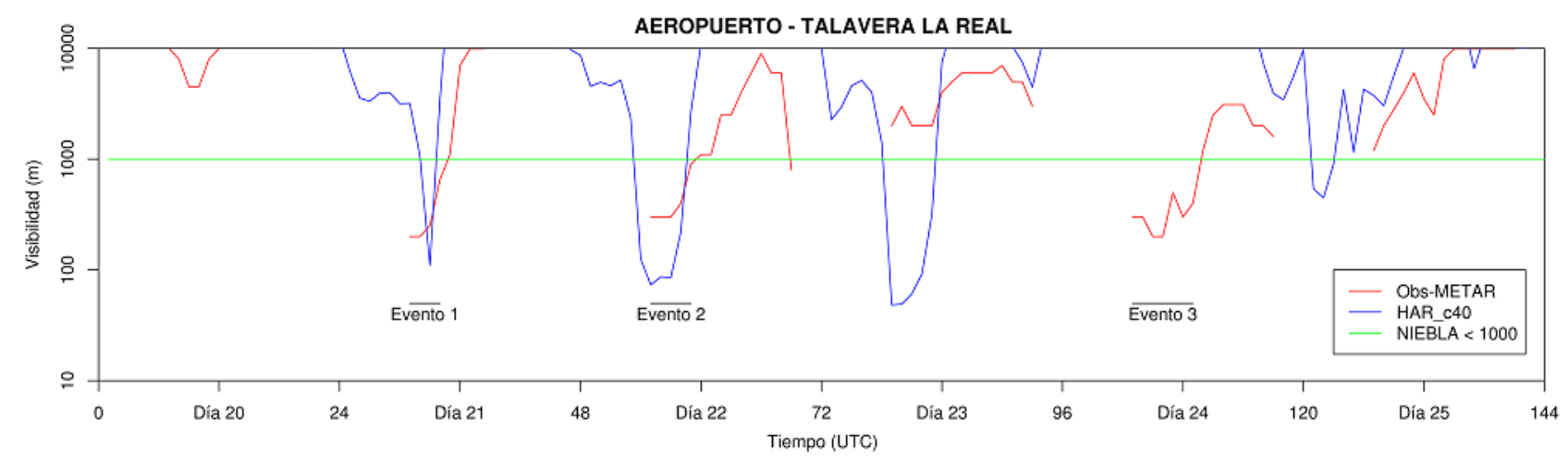

Figura 7. Ejemplo de la comparación de las observaciones de visibilidad registradas en los partes METAR del aeropuerto de Talavera la Real y los valores previstos por el modelo H-A en esa misma localización.

\subsection{Verificación de la predicción de nubes bajas y muy bajas de H-A con el producto CT del NWC SAF filtrado a nubes bajas usando el método SAL}

Para los tres eventos señalados anteriormente en la figura 7 se ha realizado el siguiente estudio de comparación espacial y temporal de los productos de satélite y los valores de H-A usando el método SAL.

Aunque dichos eventos tuvieron un comienzo, duración y finalización diferentes, se ha escogido un periodo de estudio común aproximado para los tres casos, desde las 04 UTC hasta las 14 UTC. Posteriormente, durante el análisis se especificaran cuando convenga los diferentes periodos para cada evento.

\section{2.a. Evento 1 - Día 21 de enero de 2018}

Para el primer evento de niebla se ha observado (figura 8) que la mayoría de los puntos se encuentran en el segundo cuadrante, lo que supone una sobrestimación de los parámetros tanto de la amplitud (A) como de la estructura (S), con mejores valores en la amplitud que en la estructura de los objetos. Con respecto a la localización (L) se observan valores menores de 1, lo que supone que se producen errores en la localización de los centros de masas de los objetos pero estos errores no son muy grandes, la mediana y el rango intercuartílico (IQR) del parámetro de localización se encuentran alrededor de 0,5 y no existe una gran dispersión entre los valores de los diferentes sucesos. En la figura 9 se han representado estos mismos valores con respecto al tiempo:

Con respecto a la evolución temporal de los parámetros, se observa (véase figura 9) una tendencia decreciente hacia valores más óptimos de los parámetros desde el comienzo de la niebla hasta la disipación. En este caso las nieblas comenzaron a disiparse alrededor de las 10 UTC cuando se aprecia una mejoría apreciable de los parámetros después de esa hora.

Figura 8 .

Diagrama SAL para el evento 1 (21 de enero de 2018).

Cada uno de los puntos representa un valor SAL cada 15 minutos desde las 04 UTC hasta las 14 UTC.

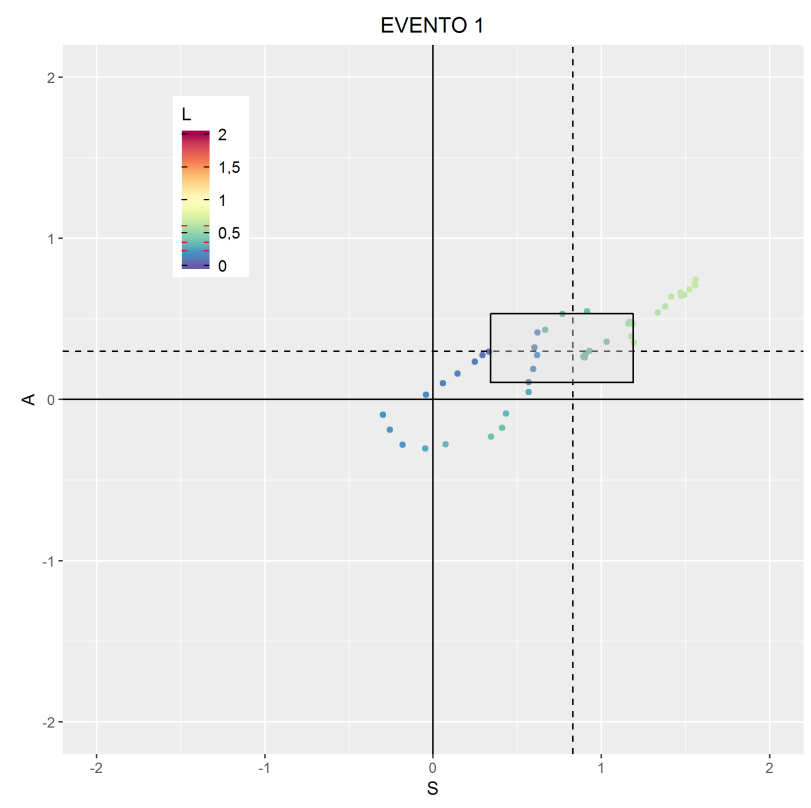


a)

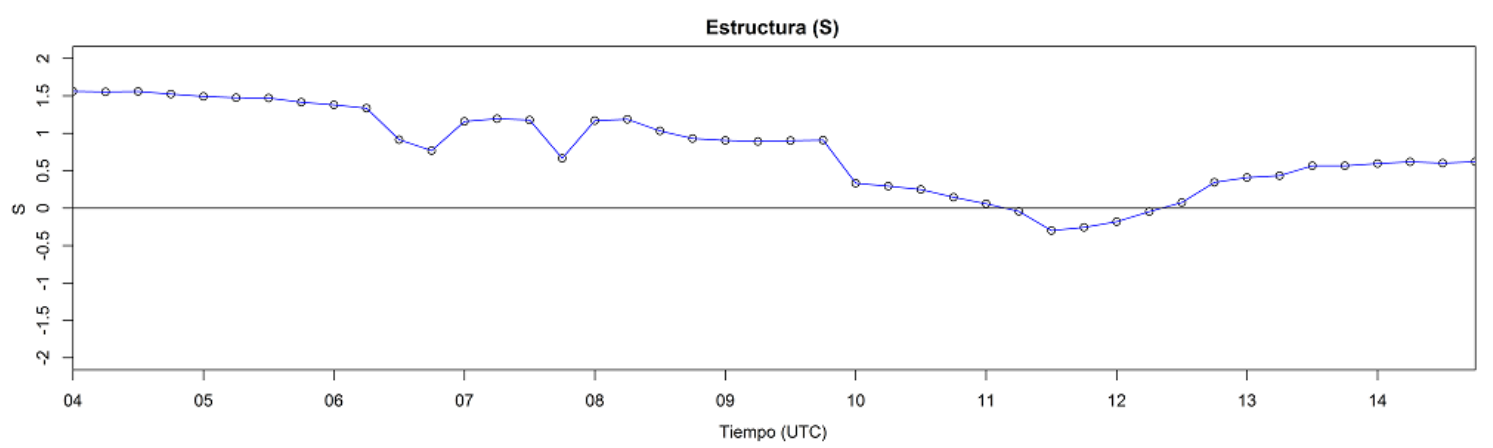

b)

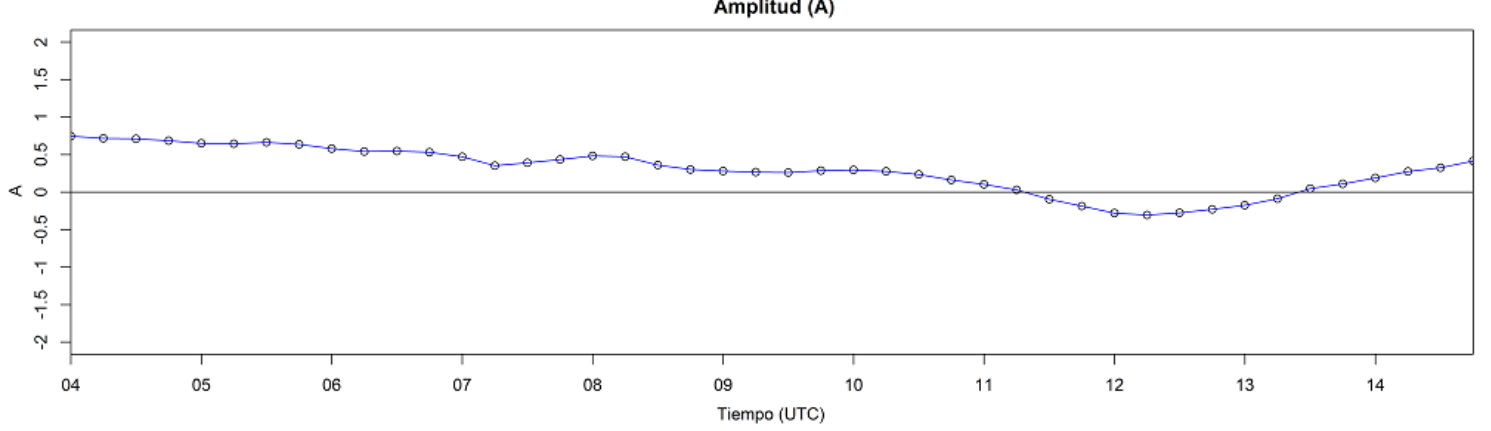

c)

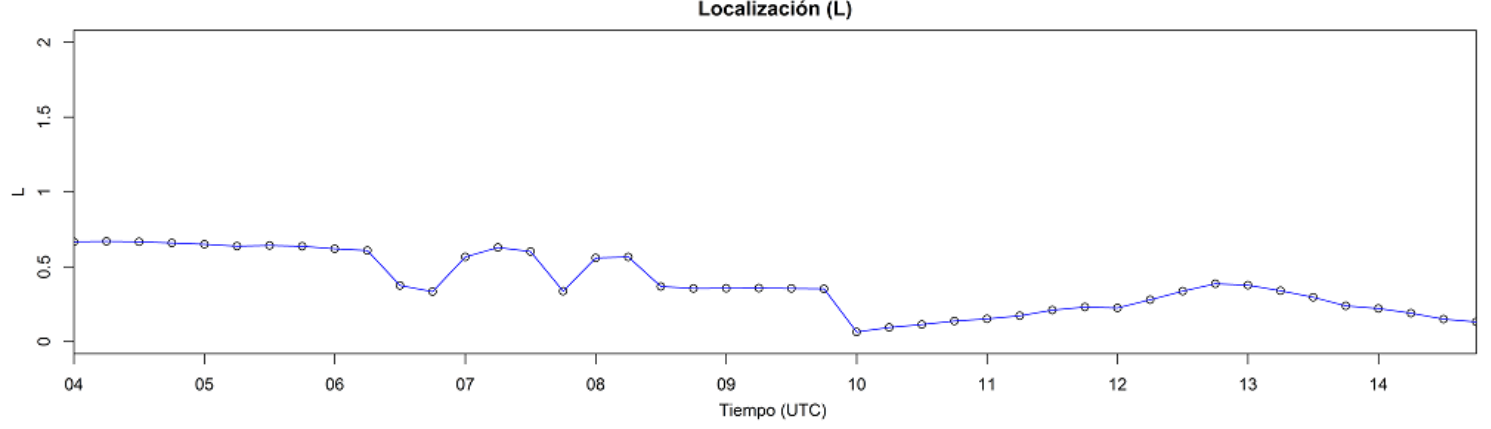

Figura 9. Representación temporal para el evento 1 de los parámetros: a) S (Estructura), b) A (Amplitud) y

c) L (Localización). Cada uno de los puntos representa un valor de SAL cada 15 minutos desde las 04 UTC hasta las 14 UTC.

\section{2.b. Evento 2 - Día 22 de enero de 2018}

En este caso, las medianas y los rangos IRQ se encuentran en el tercer y cuarto cuadrante, muy próximos a valores óptimos, indicando una pequeña subestimación de $\mathrm{S}$ y A, lo que puede significar una predicción de objetos un poco más pequeños o agudos que los observados. Con respecto al parámetro L los valores son también muy pequeños lo que sugiere una buena localización. Por tanto, para la mayoría de los casos los tres parámetros se encuentran muy cercanos a los valores ideales.

Figura 10.

Diagrama SAL para el evento 2. Cada una de los puntos representa un valor SAL cada 15 minutos desde las 04 UTC hasta las 14 UTC.

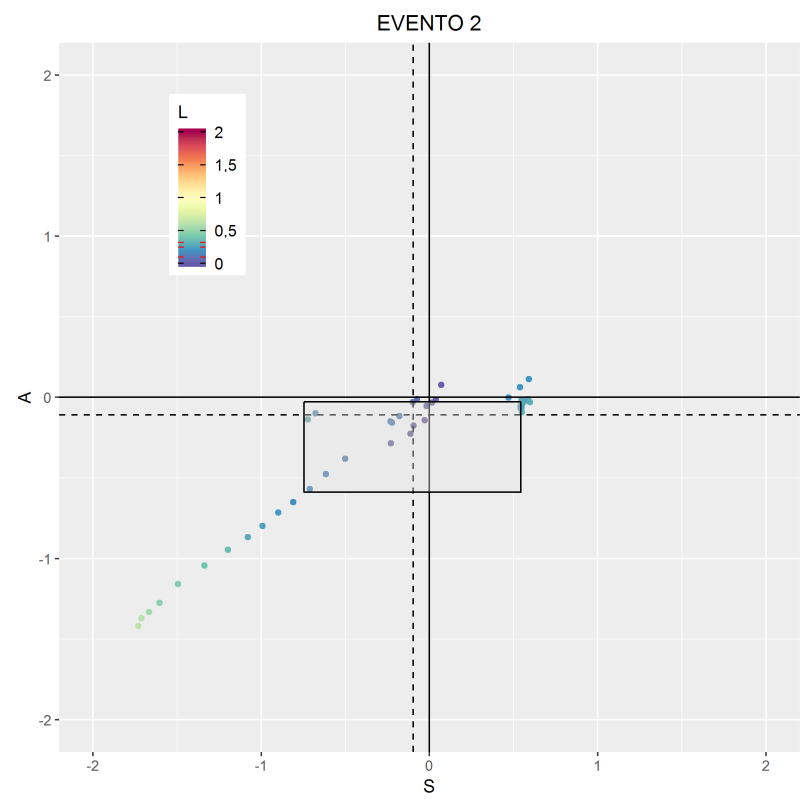


a)

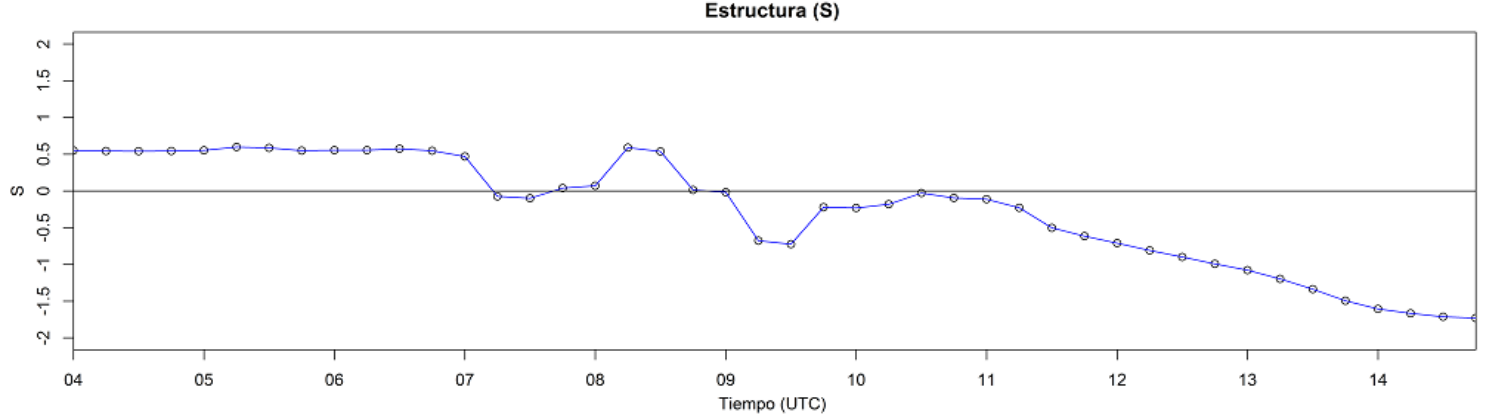

b)

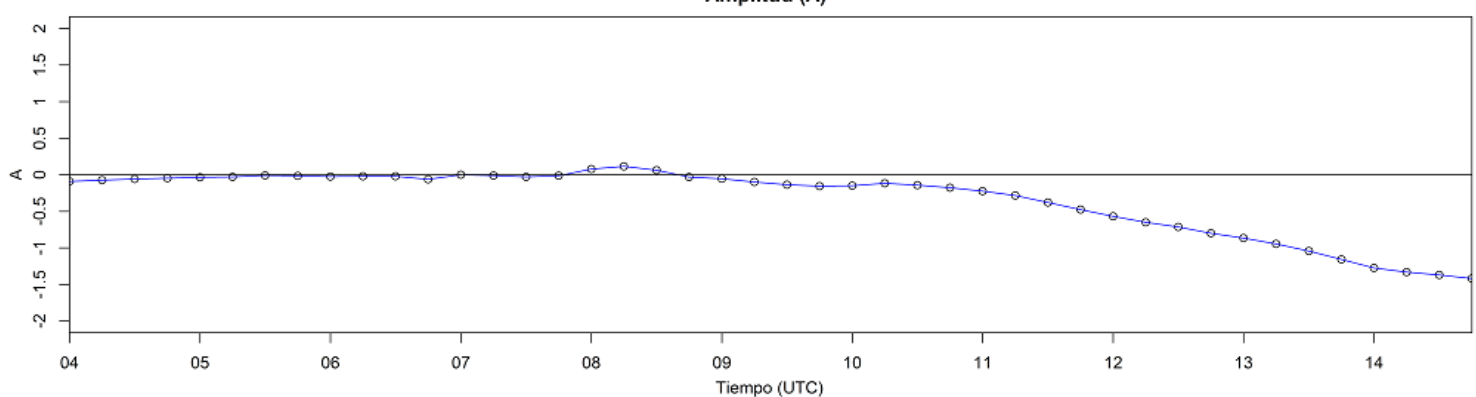

c)

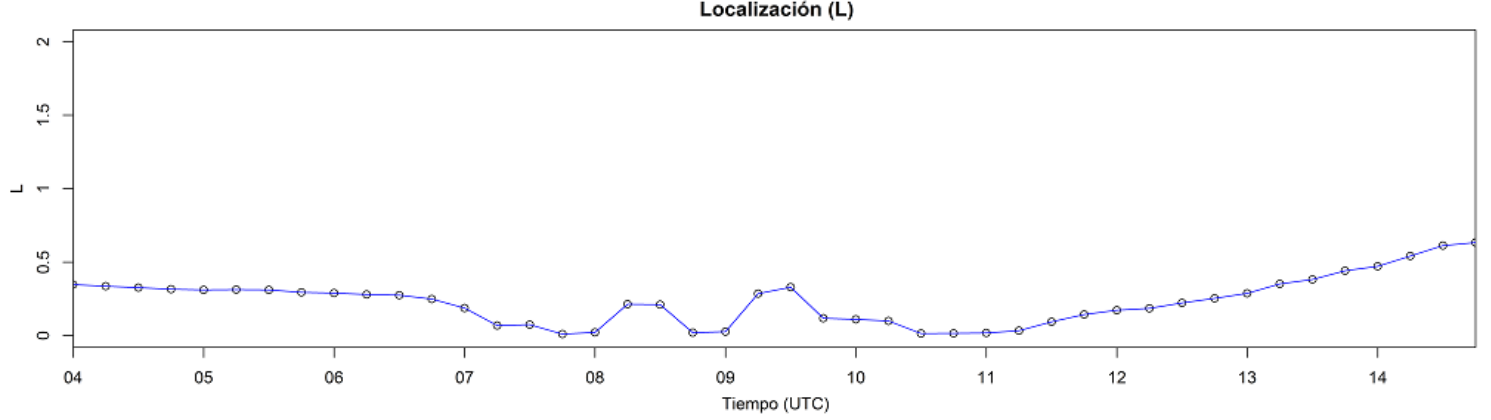

Figura 11. Representación temporal para el evento 2 de los parámetros: a) S (Estructura), b) A (Amplitud) y

c) L (Localización). Cada uno de los puntos representa un valor de SAL cada 15 minutos desde las 04 UTC hasta las 14 UTC.

En este caso, al ver su evolución temporal (figura 11) se observan valores óptimos al comienzo y durante la evolución de la niebla y una importante subestimación con respecto a los tres parámetros, estructura (S), amplitud (A) y localización (L) durante la disipación a partir de las 11 UTC.

\section{2.c. Evento 3 - Día 24 de enero de 2018}

En este último caso (figura 12), el centro de la distribución del evento se encuentra en el cuarto cuadrante, lo que significa una sobrestimación de la estructura $(\mathrm{S})$, una subestimación de la amplitud (A) y valores altos del parámetro de localización (L), con una gran dispersión (rango IQR) de los valores con respecto a $\mathrm{S}$ y a L.

Figura 12.

Diagrama SAL para el evento 3. Cada una de los puntos representa un valor SAL cada 15 minutos desde las 04 UTC hasta las 14 UTC.

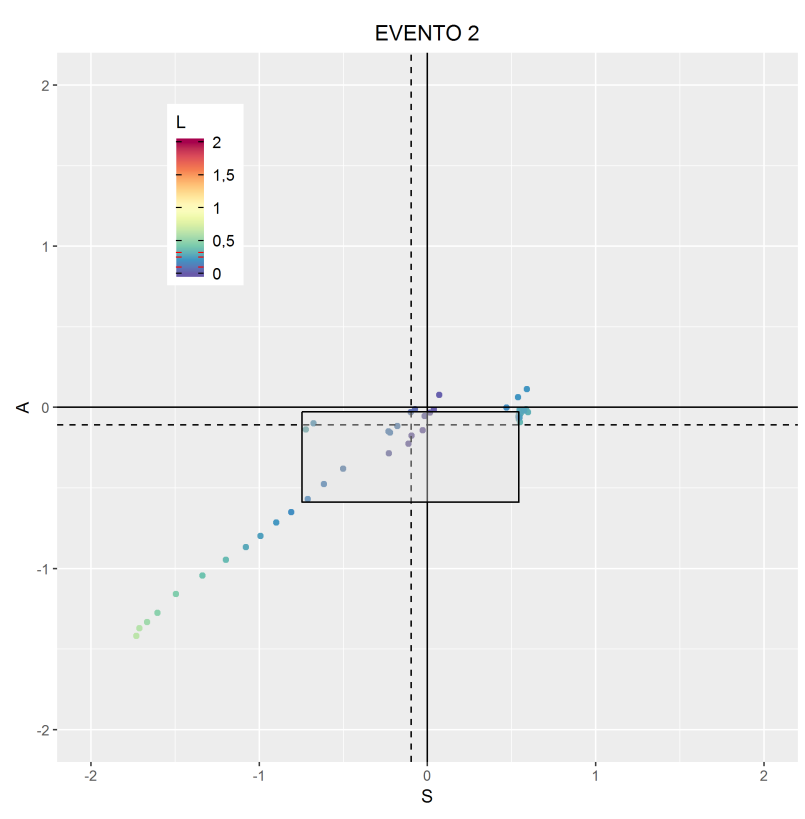


En la evolución temporal de los parámetros se observa un empeoramiento de S durante el periodo de disipación de las nieblas, para el parámetro A el empeoramiento se produce durante el desarrollo y evolución mejorando en la disipación y para el parámetro L los valores también empeoran en el periodo de disipación (figura 13).

a)

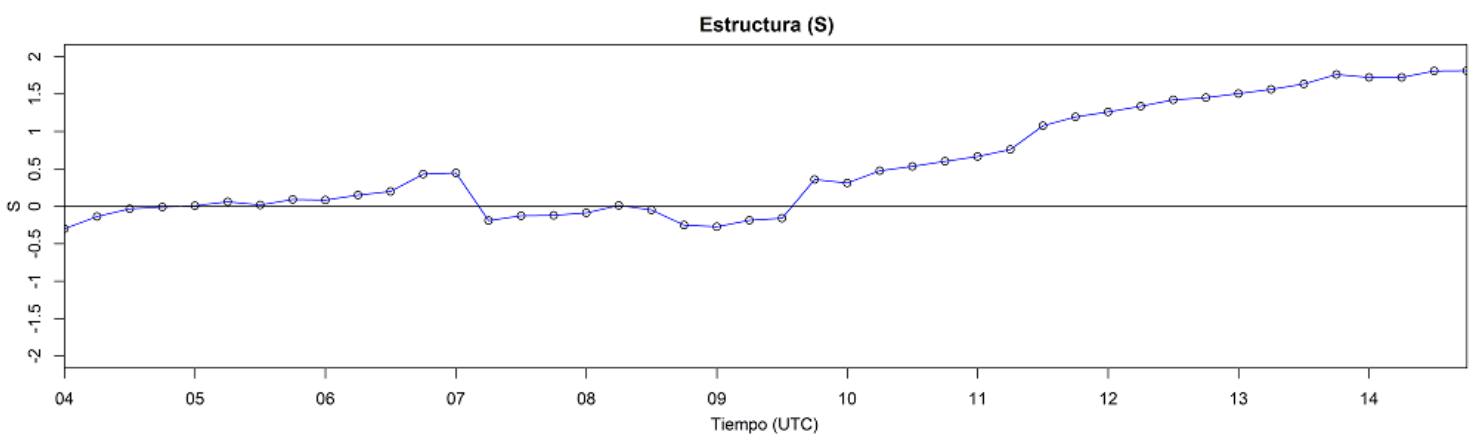

b)

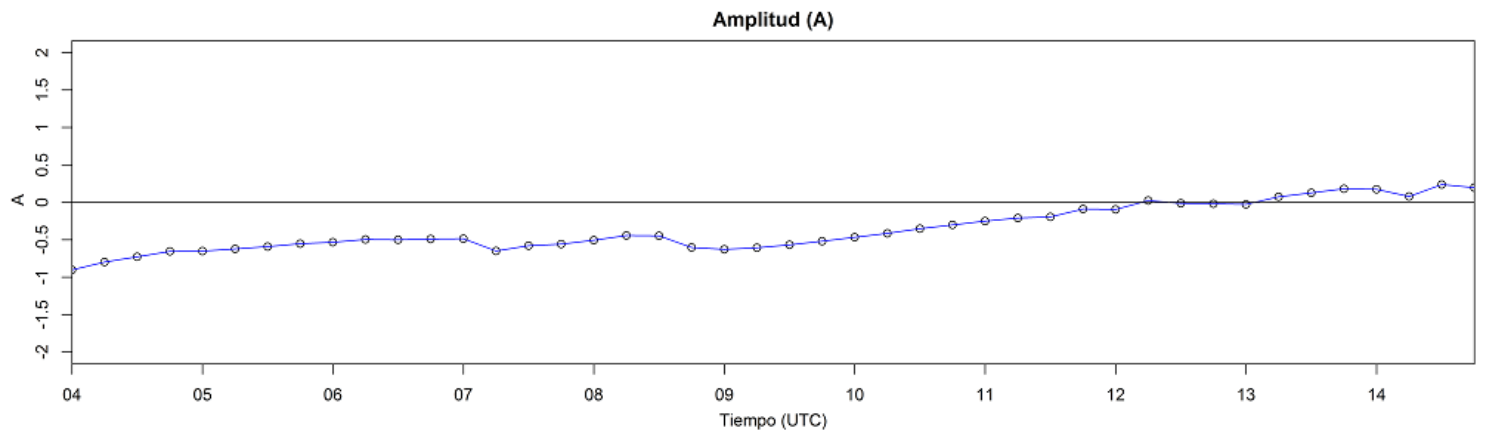

c)

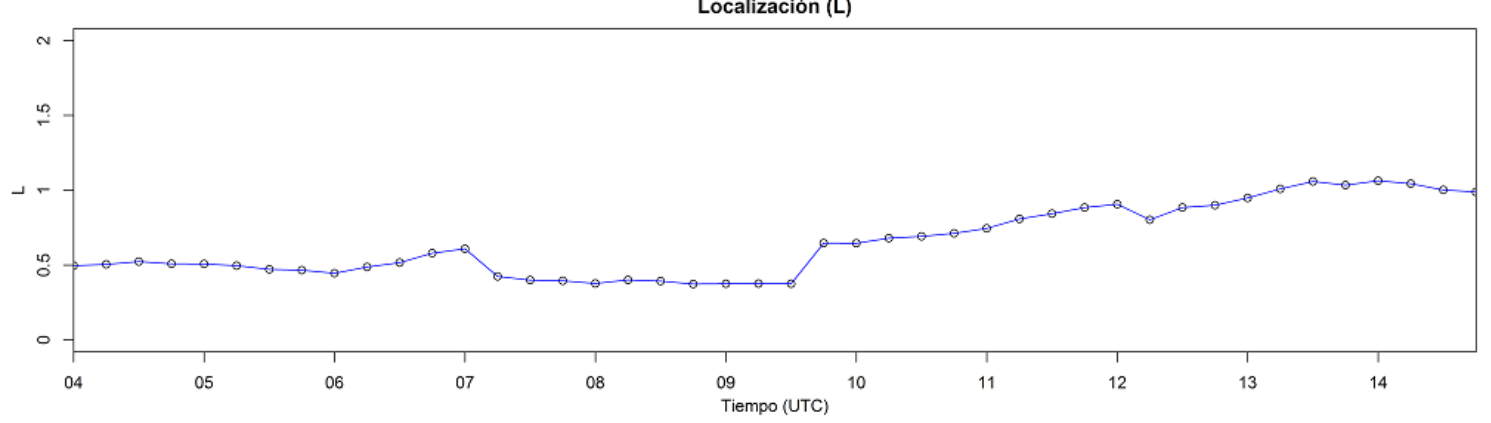

Figura 13. Representación temporal para el evento 3 de los parámetros: a) S (Estructura), b) A (Amplitud) y c) L (Localización). Cada uno de los puntos representa un valor de SAL cada 15 minutos desde las 04 UTC hasta las 14 UTC.

\section{CONCLUSIONES}

Las nieblas son procesos complejos de analizar y ya sea a través de observaciones indirectas del fenómeno o con el campo de nubes bajas la labor es de gran dificultad. Con este trabajo se ha intentado dar un pequeño paso en este camino con la comparación de las observaciones puntuales de diferentes parámetros y el producto de satélite de nubes bajas con respecto a las salidas del modelo H-A en su última versión, para unos pocos casos de estudios de nieblas desde una manera objetiva y cuantitativa.

Con la implementación y evaluación del método SAL hemos intentado dar una mayor información para la evaluación de las salidas del modelo desde diferentes perspectivas, complementado la verificación clásica. 
Con la verificación clásica se han encontrado grandes discrepancias en el parámetro de visibilidad para la mayoría de las estaciones, las cuales parecen aumentar para los casos de nieblas. En general, el acuerdo fue bueno para el resto de los parámetros analizados.

El método SAL se ha aplicado a la verificación de 3 eventos puntuales de nieblas invernales de radiación en las cuencas hidrográficas del Tajo y el Guadiana, mostrando en general buenos resultados. En particular, para el primer caso se ha encontrado una pequeña sobrestimación de las predicciones con respecto a su amplitud, estructura y localización, con una tendencia de mejora de los parámetros hasta la fase de disipación. Para el segundo caso, los parámetros se acercan mucho a los valores óptimos $(\mathrm{S}=\mathrm{A}=\mathrm{L}=0)$, y de igual modo los valores más cercanos a cero se localizan en las fases de formación y desarrollo y una subestimación de los mismos en el proceso de disipación. Por último, en el tercer caso la comparación entre las predicciones y las observaciones produce los peores resultados de los tres casos, con una sobrestimación de la estructura, subestimación de la amplitud e importantes errores en la localización de los centros de masas de los objetos, otra vez encontrando los peores valores de S y L para la fase de disipación y de A en las fases de formación y desarrollo.

A la luz de estos resultados y a pesar de las limitaciones y baja representatividad de este estudio, podemos decir que el modelo $\mathrm{H}$-A ha probado ser un buen predictor con respecto a la estructura, amplitud y localización de los procesos de nubes bajas o nieblas, con aparentes limitaciones en la fase de disipación.

Esperamos que estos resultados sean el comienzo de un estudio más detallado para la identificación de parámetros y de puntos fuertes y débiles de la predicción numérica actual en la predicción de este fenómeno y su evolución.

Para ello está planeado ampliar el estudio a un mayor número de casos (tanto en el tiempo como en el espacio) para derivar en conclusiones más sólidas, seleccionado diferentes regiones de la península ibérica con diferentes condiciones y utilizando diferentes metodologías de comparación de campos.

\section{REFERENCIAS}

Davis, C., Brown, B. y Bullock, R., 2006. Object-based verification of precipitation forecasts. Part I: Methodology and application to mesoscale rain areas. Mon. Wea. Rev., 134, 1772-1784. doi: 10.1175/ MWR3145.1.

Seity, Y., Brousseau, P., Malarde, S., Hello, G., Bénard, P., Bouttier, F., Lac, C. y Masson V., 2011. The AROME-France Convective-Scale Operational Model. Mon. Wea. Rev., 139, 976-991.

Weniger, M. y Friederichs, P., 2016. Using the SAL Technique for Spatial Verification of Cloud Processes: A Sensitivity Analysis. Journal of Applied Meteorology and Climatology, 55, 2091-2108. doi: 10.1175/ JAMC-D-15-0311.1.

Wernli, H., Paulat, M., Hagen, M. y Frei, C., 2008. SAL-A novel quality measure for the verification of quantitative precipitation forecasts. Mon. Wea. Rev., 136, 4470-4487. doi: 10.1175/2008MWR2415.1. 